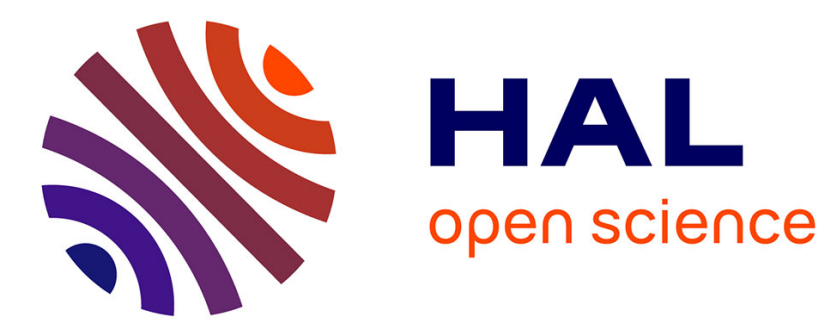

\title{
Investigating self-similarity and heavy-tailed distributions on a large scale experimental facility
}

Loiseau Patrick, Paulo Gonçalves, Guillaume Dewaele, Pierre Borgnat, Patrice Abry, Pascale Primet Vicat-Blanc

\section{- To cite this version:}

Loiseau Patrick, Paulo Gonçalves, Guillaume Dewaele, Pierre Borgnat, Patrice Abry, et al.. Investigating self-similarity and heavy-tailed distributions on a large scale experimental facility. IEEE/ACM Transactions on Networking, 2010, 99, pp.1. 10.1109/TNET.2010.2042726 . ensl-00475902

\section{HAL Id: ensl-00475902}

\section{https://hal-ens-lyon.archives-ouvertes.fr/ensl-00475902}

Submitted on 23 Apr 2010

HAL is a multi-disciplinary open access archive for the deposit and dissemination of scientific research documents, whether they are published or not. The documents may come from teaching and research institutions in France or abroad, or from public or private research centers.
L'archive ouverte pluridisciplinaire HAL, est destinée au dépôt et à la diffusion de documents scientifiques de niveau recherche, publiés ou non, émanant des établissements d'enseignement et de recherche français ou étrangers, des laboratoires publics ou privés. 


\title{
Investigating self-similarity and heavy-tailed distributions on a large scale experimental facility
}

\author{
Patrick Loiseau, Paulo Gonçalves, Member, IEEE, Guillaume Dewaele, Pierre Borgnat, Member, IEEE, Patrice \\ Abry, Senior Member, IEEE, and Pascale Vicat-Blanc Primet, Member, IEEE
}

\begin{abstract}
After the seminal work by Taqqu et al. relating selfsimilarity to heavy-tailed distributions, a number of research articles verified that aggregated Internet traffic time series show self-similarity and that Internet attributes, like Web file sizes and flow lengths, were heavy-tailed. However, the validation of the theoretical prediction relating self-similarity and heavy tails remains unsatisfactorily addressed, being investigated either using numerical or network simulations, or from uncontrolled Web traffic data. Notably, this prediction has never been conclusively verified on real networks using controlled and stationary scenarii, prescribing specific heavy-tailed distributions, and estimating confidence intervals. With this goal in mind, we use the potential and facilities offered by the large-scale, deeply reconfigurable and fully controllable experimental Grid5000 instrument, to investigate the prediction observability on real networks. To this end we organize a large number of controlled traffic circulation sessions on a nation-wide real network involving two hundred independent hosts. We use a FPGA-based measurement system, to collect the corresponding traffic at packet level. We then estimate both the self-similarity exponent of the aggregated time series and the heavy-tail index of flow size distributions, independently. On the one hand, our results complement and validate with a striking accuracy some conclusions drawn from a series of pioneer studies. On the other hand, they bring in new insights on the controversial role of certain components of real networks.
\end{abstract}

\section{Motivations}

Comprehension and prediction of network traffic is a constant and central preoccupation for Internet Service Providers. Challenging questions, such as the optimization of network resource utilization that respect the application constraints, the detection (and ideally the anticipation) of anomalies and congestion, contribute to guarantee a better quality of service $(\mathrm{QoS})$ to users. From a statistical viewpoint, this is a challenging and arduous problem that encompasses several components: network design, control mechanims, transport protocols and the nature of traffic itself. In the last decade, great attention has been devoted to the statistical study of time

Patrick Loiseau is with Université de Lyon, École Normale Supérieure de Lyon (LIP), 46 allée d'Italie, 69364 Lyon cedex 07, France. (e-mail: Patrick.Loiseau@ens-lyon.fr)

Paulo Gonçalves and Pascale Vicat-Blanc Primet are with INRIA, Université de Lyon, École Normale Supérieure de Lyon (LIP), 46 allée d'Italie, 69364 Lyon cedex 07, France. (e-mails: Paulo.Goncalves@enslyon.fr, Pascale.Primet@ens-lyon.fr)

Guillaume Dewaele is with Université de Lyon, École Normale Supérieure de Lyon, Laboratoire de Physique, 46 allée d'Italie, 69364 Lyon cedex 07, France. (e-mail: Guillaume.Dewaele@ens-lyon.fr)

Pierre Borgnat and Patrice Abry are with CNRS, UMR 5672, Université de Lyon, Laboratoire de Physique, École Normale Supérieure de Lyon, 46 allée d'Italie, 69364 Lyon cedex 07, France. (e-mails: Pierre.Borgnat@ens-lyon.fr, Patrice.Abry@ens-lyon.fr) series and random variables, which collected at the core of networks, are valuable fingerprints of the system state and of its evolution. With this in mind, the pioneering work by [1] and [2] evidenced that the Poisson hypothesis, a relevant and broadly used model for phone networks, failed at describing computer network traffic. Instead, self-similarity was shown a much more appropriate paradigm, and since then, many authors have reported its existence in a wide variety of traffics [3], [4], [5], [6]. Following up this prominent discovery, the theoretical work by Taqqu and collaborators constituted another major breakthrough in computer network traffic modeling, identifying a plausible origin of self-similarity in traffic time series [2], [7], [8]. It posits that the heavy-tail nature of some probability distributions, mainly that of flow size distributions, suffice to generate traffic exhibiting long range dependence, a particular manifestation of self-similarity [9]. To support their claim, they established a close form relation connecting the heavy-tail thickness (as measured by a tail index) and the self-similarity exponent.

Notwithstanding its mathematical soundness, pragmatic validity of this model has been corroborated with real world traffic data only partially, so far. The first pitfall lies in the definition of long range dependence itself, which, as we will see, is a scale invariance property that holds only asymptotically for long observation durations. Its consistent measurement requires that experimental conditions maintain constant, and that no external activity perturbs the traffic characteristics. In those conditions, finding a scale range that limits itself to stationary data, and that is sufficiently wide to endorse reliable self-similarity measurements, is an intricate task.

Secondly, even though real traffic traces had led to check concordance between tail index and self-similarity exponent, only was it perceived for a given network configuration that necessarily corresponded to a single particular value of the parameters set. An extensive test, to verify that self-similarity exponent obeys the same rule when the tail index is forced to range over some interval of interest, was never performed on a large scale real network platform.

Finally, the exact role of the exchange protocol, viewed as a subsidiary factor from this particular model, is still controversial [10], [11], [12]. Due to the lack of flexible, versatile, while realistic experimental environments, part of this metrology questioning has been addressed by researchers of the network community, using simulators, emulators or production platforms. However, these tools have limitations on their own, which turn difficult the studies, and yield only 
incomplete results.

In the present work, we use the potential and the facilities offered by the very large-scale, deeply reconfigurable and fully controlable experimental Grid5000 instrument [13] to empirically investigate the scope of applicability of Theorem proposed by Taqqu et al. [2], [7], [8].

Under controlled experimental conditions, we first prescribe the flow size distribution to different tail indices and compare the measured traffic self-similar exponents with their corresponding theoretical predictions. Then, we elucidate the role of the protocol and of the rate control mechanism on traffic scaling properties. In the course, we resort to efficient estimators of the heavy-tail index and of the self-similarity exponent derived from recent advances in wavelet based statistics and time series analysis. In our opinion, this revisited investigation is the missing prerequisite to a rigorous methodological approach to assess the actual applicability conditions of this theoretical bond regarding real applications. That is why we deemed important to start with an advisedly elementary and yet realistic traffic pattern, run on a real platform, under plainly controlled network configurations. The sequel is organized as follows. Section II summarizes related works. Section III elaborates on theoretical foundations of the present work, including a concise definition of parameters of interest. In Section IV we develop the specifities of our experimental testbed, and we describe our experimental designs. Section V presents and comments the results. Conclusions and perspectives are drawn in Section VI.

\section{RELATED WORK}

Without giving full bibliography on the subject (many can be found in [4], [5], [14]), there have been extensive reports on self-similarity in network traffic. As most of them are based on measurements and on analysis of real-world traces from the Internet, they only permit experimental validation of a single point on the prediction curve of Taqqu's Theorem, corresponding to one particular configuration. As its was presented before, the question here is more on the relation between these two properties. This relation is rooted in the seminal work [2], [7] about the M/G/N queueing models with heavy-tailed distributions of ON periods. Nonetheless, first experimental works by Crovella and co-authors [3], [11], hinted that this theoretical relation holds for internet traffic, and later on, also for more general types of traffic [10], [12]. However, due to the impossibility of controlling important parameters when monitoring the Internet, only compatibility of the formula could be tested against real data, but there is no statistically grounded evidences that self-similarity measured in network traffic is the work of this sole equality. On the other hand, study of self-similarity at large scales is very sensitive to inevitable non-stationnarities (day and week periodicities for instance) and to fortuitous anomalies existing on the Internet (see for instance [15]). It seems that the question has, since, never received a full experimental validation. In order to obtain such a validation, an important feature is to be able to make the heavy-tail index vary, now there is only few attempts to validate the relation under these conditions. One is conducted in [11], that uses a network simulator, and where some departure from the theoretical prediction is reported (Fig. 3 in this article). This deviation is probably caused by the limited length of the simulation and also by the bias introduced by the used scaling estimator ( $\mathrm{R} / \mathrm{S}$ and Variance-Time) on short traces. Actually, the main restriction of simulators lies in their scalability limitation, and in the difficulty of their validation. Indeed, the network is an abstraction, protocols are not production code, and the number of traffic sources or bitrates you can simulate depends on the computing power of the machine.

Large-scale experimental facilities are alternatives that may overcome both Internet and simulators limitations as they permit to control network parameters and traffic generation, including statistics and stationarity issues. Emulab [16] is a network experimental facility where network protocols and services are run in a fully controlled and centralized environment. The emulation software runs on a cluster where nodes can be configured to emulate network links. In an Emulab experiment, the user specifies an arbitrary network topology, having a controllable, predictable, and reproducible environment. He has full root access on PC nodes, and he can run the operating system of his choice. However, the core network's equipments and links are emulated. The RON testbed [17] consists of about 40 machines scattered around the Internet. These nodes are used for measurement studies and evaluation of distributed systems. RON does not offer any reconfiguration capability at the network or at the nodes' level. The PlanetLab testbed [18] consists of about 800 PCs on 400 sites (every site runs 2 PCs) connected to the Internet (no specific or dedicated link). PlanetLab allows researchers to run experiments under real-world conditions, and at a very large scale. Research groups are able to request a PlanetLab slice (virtual machine) in which they can run their own experiment.

Grid5000 [13], the experimental facility we use in the present work, proposes a different approach where the geographically distributed resources (large clusters connected by ultra high end optical networks) are running actual pieces of software in a real wide area environment. Grid5000 allows reproducing experimental conditions, including network traffic and CPU usage. This feature warrants that evaluations and comparisons are conducted according to a strict and scientific method. Grid5000 proposes a complimentary approach to PlanetLab, both in terms of resources and of experimental environment.

\section{THEORY}

Taqqu's Theorem relates two statistical properties that are ubiquitously observed in computer networks: On the one hand, self-similarity that is defined at the level of aggregated timeseries of the traffic, and on the other hand, heavy-tailness that involves grouping of packets. Simplistically, network traffic is described as a superposition of flows (without notions of users, or sessions,...) that permits us to adopt the following simple two-level model: (i) Packets are emitted and grouped in flows whose length (or number of packets) follows a heavytailed distributed random variable [19], [20], [21]; (ii) the sum 
over those flows approximates network traffic on a link or a router. This crude description is coherent with current (yet more elaborate) statistical model for Internet traffic [20], [21].

After a succinct definition of these two statistical properties, we present the corresponding parameter estimation procedures that we use in our simulations, and chosen amongst those reckoned to present excellent estimation performance.

\section{A. Self-similarity and long range dependence}

1) Definition: Taqqu's Theorem implies that Internet time series are relevantly modeled by fractional Brownian motion (fBm), the most prominent member of a class of stochastic processes, referred to as self-similar processes with stationary increments ( $H$-sssi, in short). Process $X$ is said to be $H$-sssi if and only if it satisfies [9]:

$$
\begin{aligned}
X(t)-X(0) & \stackrel{f d d}{=} X(u+t)-X(u), \forall t, u \in \mathbb{R}, \\
X(t) & \stackrel{f d d}{=} a^{H} X\left(\frac{t}{a}\right), \forall t, a>0,0<H<1,
\end{aligned}
$$

where $\stackrel{f d d}{=}$ means equality for all finite dimensional distributions. Eq. (1) indicates that the increments of $X$ form stationary processes (while $X$ itself is not stationary). Essentially, self-similarity, Eq. (2), means that no characteristic scale of time can be identified as playing a specific role in the analysis or description of $X$. Corollarily, Eq. (2) implies that $\mathbb{E} X(t)^{2}=\mathbb{E} X(1)^{2} t^{2 H}$, underlining both the scale free and the non-stationary natures of the process.

It turns out that the covariance function of the increment process, $Y(t)=X(t+1)-X(t)$, of a $H$-sssi process $X$ satisfies, for $|\tau| \rightarrow+\infty$ :

$$
\mathbb{E} Y(t) Y(t+\tau) \sim \mathbb{E} X(1)^{2} H(2 H-1)|\tau|^{2 H-2},
$$

When $1 / 2<H<1$, hence $0<2-2 H<1$, such a power law decay of the covariance function of a stationary process is referred to as long range dependence [9], [14].

Long range dependence and self-similarity designate two different notions, albeit often confused. The latter is associated to non stationary time series, such as fBms, while the former is related to stationary time series, such as fBm's increments. In the present work, given that Taqqu's Theorem predicts that the cumulated sums of aggregated Internet time series are selfsimilar, we adopt here the same angle and discuss the results in terms of self-similarity of the integrated traces.

2) Self-similarity parameter estimation: In [22], it was shown that wavelet transforms provide a relevant procedure for the estimation of the self-similarity parameter. This procedure revealed particularly efficient at analyzing Internet time series in [5], [6] and has then been massively used in this context.

Let $d_{X}(j, k)=\left\langle\psi_{j, k}, X\right\rangle$ denote the (Discrete) Wavelet Transform coefficients, where the collection $\left\{\psi_{j, k}(t)=\right.$ $\left.2^{-j / 2} \psi_{0}\left(2^{-j} t-k\right), k \in \mathbb{Z}, j \in \mathbb{Z}\right\}$ forms a basis of $L^{2}(\mathbb{R})$ [23]. The reference template $\psi_{0}$ is termed mother-wavelet and is characterized by its number of vanishing moments $N_{\psi}>1$, an integer such that $\int t^{k} \psi_{0}(t) d t \equiv 0, \forall k=0, \ldots, N_{\psi}-1$. Then, decomposing a $H$-sssi process, the variance of the wavelet coefficients verifies [22]:

$$
\mathbb{E}\left|d_{X}(j, k)\right|^{2}=\mathbb{E}\left|d_{X}(0,0)\right|^{2} 2^{j(2 H+1)},
$$

and, provided $N>H+1 / 2$, the sequence $\left\{d_{X}(j, k), k=\right.$ $\ldots,-1,0,1, \ldots\}$ form a stationary and weakly correlated time series [6]. These two central properties warrant to use the empirical mean $S(j)=n_{j}^{-1} \sum_{k}\left|d_{X}(j, k)\right|^{2}, \quad\left(n_{j}\right.$ being the number of available coefficients at scale $2^{j}$ ) to estimate the ensemble average $\mathbb{E}\left|d_{X}(j, k)\right|^{2}$. Eq. (4) indicates that selfsimilarity transposes to a linear behavior of $\log _{2} S(j)$ vs. $\log _{2} 2^{j}=j$ plots, often referred to as Logscale Diagrams (LD) in the literature [5], [6]. A (weighted) linear regression of the LD within a proper range of octaves $j_{1}, j_{2}$ is used to estimate $H$.

In [5], [6], [22], the estimators performance are both theoretically and practically quantified, and are proved to compare satisfactorily against the best parametric techniques. Moreover, this estimator is endowed with a practical robustness that comes from its extra degree of freedom $N_{\psi}$. In practice, the main difficulty lies in the correct choice of the regression range $j_{1} \leq j \leq j_{2}$. This will be discussed in Section $\mathrm{V}$, in the light of actual measurements.

\section{B. Heavy Tail}

1) Definition: A (positive) random variable $w$ is said to be heavy-tailed, with tail exponent $\alpha>0$ (and noted $\alpha$-HT) when the tail of its cumulative distribution function, $F_{\mathbf{w}}$, is characterized by an algebraic decrease [24]:

$$
P(\mathbf{w}>w)=1-F_{\mathbf{w}}(w) \sim L(w) \cdot w^{-\alpha} \text { for } w \rightarrow \infty,
$$

where $L(w)$ is a slowly varying function (i.e. $\forall a>$ $0, L(a w) / L(w) \rightarrow w \rightarrow \infty 1)$. A $\alpha$-HT random variable $\mathbf{w}$ has finite moments up to order $\alpha$. For instance, when $1<\alpha<2$, w has finite mean but infinite variance. A paradigm for $\alpha$-HT positive random variable is given by the Pareto distribution:

$$
F_{\mathbf{w}}(w)=1-\left(\frac{k}{w+k}\right)^{\alpha},
$$

with $k>0$ and $\alpha>1$. Its mean reads: $\mathbb{E} \mathbf{w}=k /(\alpha-1)$.

2) Tail exponent estimation: Estimation of the tail exponent $\alpha$ for $\alpha$-HT random variables is an intricate issue that received considerable theoretical attention in the statistics literature: measuring the tail exponent of a heavy-tailed distribution amounts to evaluate from observations, how fast does the probability of rare events decrease in Eq. (5). Once random variables are know to be drawn from an a priori distribution, such as the Pareto form (6) for example, parametric estimators exist and yield accurate estimates of the tail index $\alpha$ (see e.g. [25]). However, if the actual distribution of observations does not match the a priori expected $\alpha$-HT model, parametric estimators fail at measuring the tail decay.

For this reason, the non-parametric empirical estimator of $\alpha$ proposed in [26] will be preferred. The principle of this estimator is simple and relies on the Fourier mapping between the cumulative distribution function $F_{\mathbf{w}}(w)$ and the characteristic function $\chi_{\mathbf{w}}(s)$ of a random variable:

$$
\chi_{\mathbf{w}}(s)=\int e^{-i s w} d F_{\mathbf{w}}(w) .
$$


By a duality argument, the tail exponent $\alpha$ that bounds the order of finite moments of $F_{\mathbf{w}}$,

$$
\alpha=\sup _{r}\left\{r>0: \int|w|^{r} d F_{\mathbf{w}}(w)<\infty\right\},
$$

transposes to the local Lipschitz regularity of the characteristic function $\chi_{\mathbf{w}}$ at the origin, according to:

$$
\alpha=\sup _{r}\left\{r>0: 1-\Re \chi_{\mathbf{w}}(s)=\mathcal{O}\left(s^{r}\right) \text { as } s \rightarrow 0^{+}\right\},
$$

where $\Re$ stands for the real part. It is easy to recognize in this power law behavior of $\Re \chi_{\mathbf{w}}(s)$, a scale invariance property of the same type of that of relation (3), which is conveniently identifiable with wavelet analyses. Hence, computing the discrete wavelet decomposition of $\Re_{\chi_{\mathbf{w}}}$, and retaining only the wavelet coefficients that lie at the origin $k=0$, yields the following multiresolution quantity:

$$
d_{\chi_{\mathbf{w}}}(j, 0)=\mathbb{E} \Psi_{0}\left(2^{j} \mathbf{w}\right) \leq C 2^{j \alpha} \text { for } j \rightarrow-\infty,
$$

where $\Psi_{0}(\cdot)$ denotes the Fourier transform of analyzing wavelet $\psi_{0}(\cdot)$. Now, let $\left\{w_{0}, \cdots, w_{n-1}\right\}$ be a set of i.i.d. $\alpha$ $\mathrm{HT}$ random variables, and replace the ensemble average in Eq. (10) by its empirical estimator, the estimate $\widehat{\alpha}$ simply results from a linear regression of the form

$$
\begin{aligned}
\log \widehat{d}_{\chi_{\mathbf{w}}}^{(n)}(j, 0) & =\log n^{-1} \sum_{i=o}^{n-1} \Psi\left(2^{j} w_{i}\right) \\
& \approx \widehat{\alpha} j+\log C, \text { as } j \rightarrow-\infty
\end{aligned}
$$

The estimator was proved to converge for all heavy-tailed distributions, and also it has a reduced variance of estimation in $\mathcal{O}\left(n^{-1}\right)$, where $n$ is the sample size. We refer the interested reader to [26] where robustness and effective use of this estimator are thoroughly studied. Yet, let us mention the existence of a theoretical scale range where the linear model, Eq. (11), holds, and which shows very helpful for practitioners to adequately adjust their linear fitting over a correct scale range.

\section{Taqqu's Theorem}

A central result for interpreting statistical modeling of network traffic is a celebrated Theorem due to M. Taqqu and collaborators [2], [7], [8], in which heavy-tailness of flow sessions has been put forward as a possible explanation for the occurrence of self-similarity of Internet traffic.

The original result considers a $\mathrm{M} / \mathrm{G} / \mathrm{N}$ queueing model served by $N$ independent sources, whose activities $Z_{i}(t)$, $i \in\{1, \ldots, N\}$, are described as binary ON/OFF processes. The durations of the $\mathrm{ON}$ periods (corresponding to a packet train emission by a source) consists of i.i.d. positive random variables $\tau_{\mathrm{ON}}$, distributed according to a heavy-tail law $P_{\mathrm{ON}}$, with exponent $\alpha=\alpha_{\mathrm{ON}}$. Intertwined with the ON periods, the OFF periods (a source does not emit traffic), have i.i.d. random durations $\tau_{\mathrm{OFF}}$ drawn from another possibly heavytailed distribution $P_{\mathrm{OFF}}$ with tail index $\alpha=\alpha_{\mathrm{OFF}}$. Thus, the $Z_{i}(t)$ consist of a $0 / 1$ reward-renewal processes with i.i.d. activation periods.
Now, let $Y_{N}(t)=\sum_{i=1}^{N} Z_{i}(t)$ denote the aggregated traffic time series and define the cumulative process $X_{N}(T t)$ :

$$
X_{N}(t T)=\int_{0}^{T t} Y_{N}(u) \mathrm{d} u=\int_{0}^{T t}\left(\sum_{i=1}^{N} Z_{i}(u)\right) \mathrm{d} u .
$$

Taqqu's Theorem (cf. [7]) states that when taking the limits $N \rightarrow \infty$ (infinitely many users) and $T \rightarrow \infty$ (infinitely long observation duration), in this order, then $X_{N}(t T)$ behaves as:

$$
X_{N}(t T) \sim \frac{\mathbb{E} \tau_{\mathrm{ON}}}{\mathbb{E} \tau_{\mathrm{ON}}+\mathbb{E} \tau_{\mathrm{OFF}}} N T t+C \sqrt{N} T^{H} B_{H}(t) .
$$

In this relation, $C$ is a constant and $B_{H}$ denotes a fractional Brownian motion with Hurst parameter:

$$
H=\frac{3-\alpha^{*}}{2}, \text { where } \alpha^{*}=\min \left(\alpha_{\mathrm{ON}}, \alpha_{\mathrm{OFF}}, 2\right) .
$$

The order of the limits is compelling to obtain this asymptotic behavior; this has been discussed theoretically elsewhere and is beyond the issues we address here. The main conclusion of Taqqu's Theorem is that, in the limit of (infinitely) long observations, fractional Brownian motions superimposed to a deterministic linear trend, are relevant asymptotic models to describe the cumulated sum of aggregated traffic time series. Moreover, Eq. (14) shows that only heavy-tailed distributions with infinite variance (i.e., $1<\min \left(\alpha_{\mathrm{ON}}, \alpha_{\mathrm{OFF}}\right)<2$ ) can generate self-similarity associated to long range dependence (i.e. $H>1 / 2$ ). Conversely, when both activity and inactivity periods have finite variance durations, $\alpha^{*}=2$ and consequently $H=1 / 2$, which means no long range dependency.

\section{EXPERIMENTAL SETUP}

To study the practical validity of Taqqu's result, we use the potential and facilities offered by the very large-scale, deeply reconfigurable and fully controllable experimental Grid5000 instrument, so as to overcome the limitations previously exposed of emulations, simulations or measurements in production networks. Moreover, we deliberately chose to work with the simplest network configurations that permit a thorough investigation of the Taqqu's Theorem: Namely, an elementary network topology encompassing multiples independent sources whose throughputs aggregate on a single hop, and a traffic generation without congestion. This way, we isolate the nature of the flows' distributions as the main entry for Taqqu's relation. After a general overview of Grid5000, the metrology platform is then described. Design of a large set of experiments, aimed at studying the actual dependence between the network traffic self-similarity and the heavy-tailness of flow size distributions, is finally detailed.

\section{A. Grid5000 instrument overview}

Grid5000, is a 5000 CPUs nation-wide Grid infrastructure for research in Grid computing [13], providing a scientific tool for computer scientists similar to the large-scale instruments used by physicists, astronomers, and biologists. It is a research tool featured with deep reconfiguration, control and monitoring capabilities designed for studying large-scale distributed systems and for complementing theoretical models 
and simulators. Up to 17 french laboratories involved and 9 sites are hosting one or more cluster of about 500 cores each. A dedicated private optical networking infrastructure, provided by RENATER, the French National Research and Education Network is interconnecting the Grid5000 sites. Two international interconnections are also available: one at $10 \mathrm{~Gb} / \mathrm{s}$ interconnecting Grid5000 with DAS3 in the Netherlands and one at $1 \mathrm{~Gb} / \mathrm{s}$ with Naregi in Japan. In the Grid5000 platform, the network backbone is composed of private $10 \mathrm{~Gb} / \mathrm{s}$ Ethernet links connected to a DWDM core with dedicated $10 \mathrm{~Gb} / \mathrm{s}$ lambdas with bottlenecks at $1 \mathrm{~Gb} / \mathrm{s}$ in Lyon and Bordeaux (see Figure 1).

Grid5000 offers to every user full control of the requested experimental resources. It uses dedicated network links between sites, allows users to reserve the same set of resources across successive experiments, to run their experiments in dedicated nodes (obtained by reservation) and it permits users to install and to run their own experimental condition injectors and measurement software. Grid5000 exposes two tools to implement these features: OAR is a reservation tool, and Kadeploy an environment deployment system. OAR offers an accurate reservation capability (CPU/Core/Switch reservation) and integrates the kadeploy system. With Kadeploy, each user can make his own environment and have a total control on the reserved resources. For instance, software and kernel modules for rate limitation, QoS mechanisms, congestion control variants can be deployed automatically within the native operating system of a large number of communicating nodes. OAR also permits users to reserve equipements for several hours. As a consequence, Grid5000 enables researchers to run successive experiments reproducing the exact experimental conditions several times, an almost impossible task with shared and uncontrolled networks. This insures also large-duration observation windows under stationary conditions - something that is unachievable on the Internet. As a private testbed, Grid5000 turns the installation of experimental hardware, like for instance the traffic capture instrument at representative traffic aggregation points, quite easy.

\section{B. Metrology platform}

Using the facilities offered by Grid5000, a platform for metrology has been designed, and schematized in Fig. 2. Before describing the monitoring facilities and the developed data processing softwares, let us present the effective topology used for this experiment.

1) Experimental system description: Unless explicitly mentioned, all our experiments consist in producing data flow transfers between many independent client nodes (sources) and many independent server nodes (destinations). It is a classical dumbbell (or butterfly) topology with a single bottleneck of capacity, here of $C=1 \mathrm{~Gb} / \mathrm{s}$. We selected $N=100$ nodes that are able to send up to at $C_{a}=1 \mathrm{~Gb} / \mathrm{s}$ on each direction (see Fig. 2)

For our experiments, we used nodes on the Grid5000 clusters of Lyon (clients) and Rennes (servers). The average $R T T$ is then stable and equal to $12 \mathrm{~ms}$, which gives a bandwidth-delay product of 1.5 MBytes. In our forthcoming

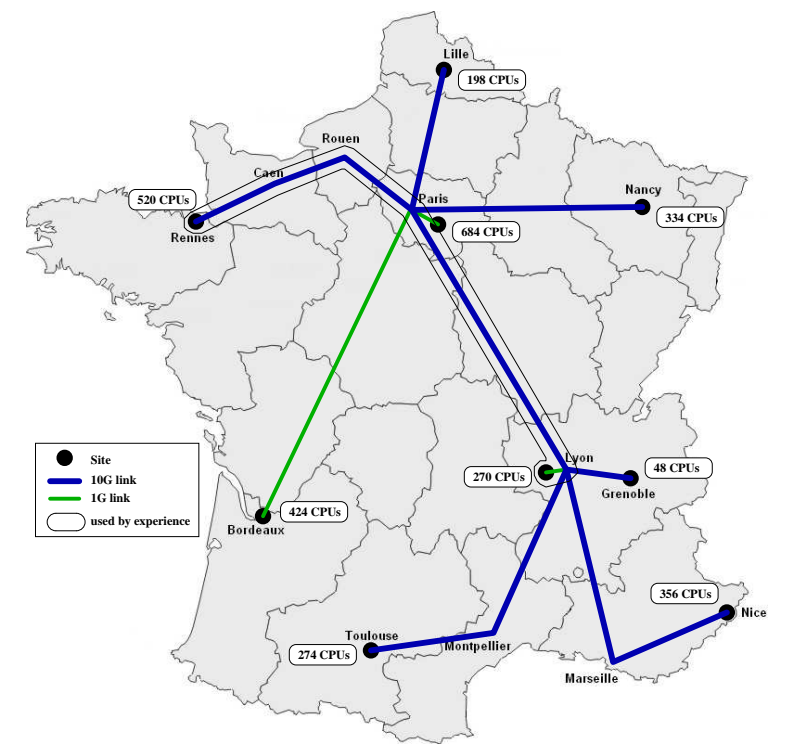

Fig. 1. Grid5000 backbone

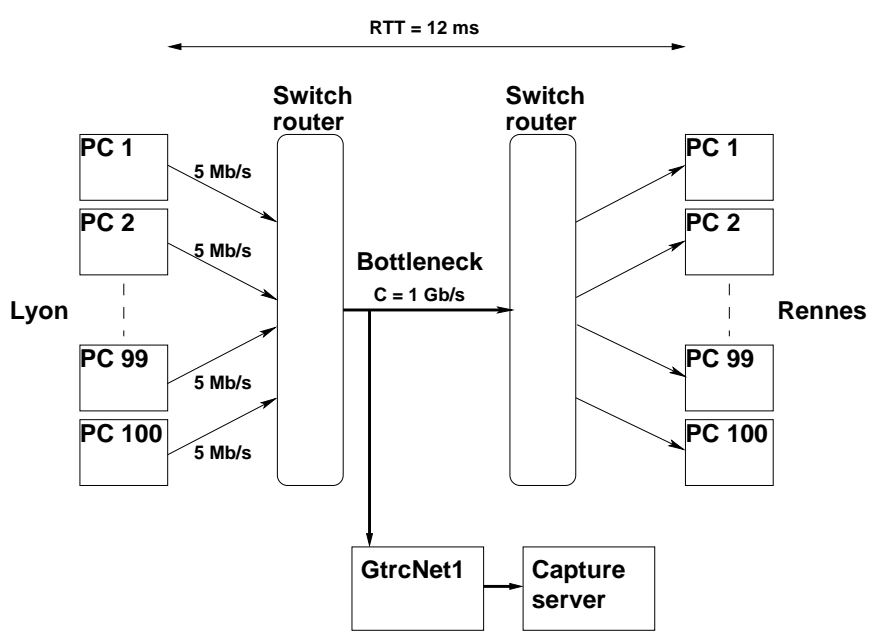

Fig. 2. Metrology platform overview

scenarii, TCP and UDP transfers are realized by using iperf [27] on Sun Fire V20z (bi-opteron) workstations of Grid5000 [13], running GNU/Linux 2.6.18.3 kernels with standard TCP and UDP modules. Iperf is a traffic generation tool that allows users to tune the different TCP and UDP parameters and to evaluate their impact on network performance.

This single bottleneck topology was intentionally chosen as our main goal was not to study the queueing effect of successive routers in a multiple hops network. We believe that, without loss of generality, the simplicity of this test topology suffices at properly creating the realistic experimental conditions for investigating the limits of Taqqu's Theorem.

2) Capture system: To measure the traffic at packet-level, we designed a specific system combining packet capture, header extraction and dedicated data analysis software. Packets are first captured by mirroring the traffic of the access link connecting the Lyon site to the rest of Grid5000. Only the outgoing traffic from the Lyon site to Grid5000 is mirrored, 
connecting a $1 \mathrm{~Gb} / \mathrm{s}$ fiber port to a $1 \mathrm{~Gb} / \mathrm{s}$ copper port directed to the monitoring system.

This system is composed of a GtrcNET-1 device [28], developed by AIST GTRC, and based on an FPGA that has been programmed to extract and aggregate packet headers and send them to an attached server. This header aggregation reduces the number of interrupts of the computer that receive the traffic to analyse, decreasing the local loss probability. In the packet capture system, the GtrcNET-1 is configured to extract a 52-Bytes headers (composed of 14, 20 and 18 Bytes of Ethernet, IP and TCP headers respectively) from the packets arriving at the one gigabit port. Headers are added a time-stamp each, encapsulated by groups of 25 into a UDP packet and then sent to another gigabit port. Time-stamps have a $60 \mathrm{~ns}\left(2^{-24} \mathrm{~s}\right)$ resolution.

The concatenated headers are stored in a computer with a quad core processor running at $2.66 \mathrm{GHz}, 4 \mathrm{~GB}$ memory, 2 ethernet gigabit ports, 300 GB SAS disk for the system, 1 RAID controller with $5 \times 300$ GB SAS disk in a RAID 0 array offering $1500 \mathrm{~GB}$ available for storing capture files. We developed a driver that reads GtrcNET-1 packets, deencapsulates time-stamped packet headers and writes them to a file in the pcap format.

3) Data processing and Flow Reconstruction: We use a series of tools over the captured IP traffic traces, to go from the packet-level traces to the aggregated traffic and the flow statistics that are needed in this work. A first step is to handle the captured IP traffic traces; secondly, we reconstruct the flows from the packets ${ }^{1}$.

IP traffic traces, saved in standard pcap format by the capture device, are first processed by ipsumdump, a program developped at UCLA [29], able to read the pcap format and to summarizes TCP/IP dump files into a self-describing binary format. Thanks to this tool, we retrieve from our traces the needed informations: time-stamps, source and destination IPs, port numbers, protocol, payload size, TCP flags, and sequence numbers. The informations are condensed into a binary file that is easier to parse, and which doesn't depend on specific capture hardware anymore.

Secondly, we have developed a collection of tools working on the ipsumdump binary format directly, which performs a variety of useful data operations on the traces. Of relevant interest here are: computation of the aggregated traffic time series (used for self-similarity estimation); extraction of traffic sub-traces for conditioned study, based on flows or packets random sampling, or on parameters filtering (traffic from/to a list of IPs, traffic on given ports, traffic using a specific protocol, etc); and reconstruction of the flows existing in the traces.

The question of flow reconstruction is an intricate problem, that is an important and difficult aspect when one wants to study the impact of their heavy-tailness [14], [30], [31], [32]. It is necessary to recompose each flow from the intertwined packets stream measured on an aggregated link. This means we must identify and group all the packets pertaining to the

\footnotetext{
${ }^{1}$ Using standard flow monitoring tools, such as Netflow or Sflow, would not be sufficient here. Indeed, we need statistical characterization at both packet-level (for $H$-sssi) and flow-level (for $\alpha$-HT).
}

same set, while considering a significantly large number of flows to guarantee statistical soundness. This constraint faces the arduous issue of loss free capture, and that of dynamic table updating.

In our tool, flows are classically defined as a set of packets that share the same 5-uplet comprising: source and destination IPs, ports, and protocol. However, because there is a finite number of ports, it is possible for two different flows to share the same 5-uplet, and thus to get grouped in a single flow. To avoid this, we set a timeout threshold: a flow is considered as finished, if its packet train undergoes an interruption lasting more that timeout. Any subsequent packet with the same 5uplet will tag the beginning of a new flow. Naturally, a proper choice of timeout is delicate, but that is the only solution that works for any kind of flows. For TCP flows, though, things are easier, as we can use the SYN or SYN/ACK flags to initiate a flow (closing any currently open flow with the same 5-uplet), and the FIN or RCT flag to close the flow, dispensing with timeout. Note that timeout remains necessary when the FIN packet is accidently missing.

Flow reconstruction (with timeout) is then performed in a table that contains all currently open flows, using hash functions to speed up the access. The relatively modest trace bitrate allows for keeping the whole table in memory. Since TCP sequence numbers and payload size for TCP packets are captured, it is possible to search for dropped or re-emitted packets during the flow reconstruction and take that into account. Elementary statistics on the flows are then available: number of packets, number of Bytes, duration of the flow, etc.

All together the data processing tools extract the two elements needed for this study: the aggregated time-series at packet-level, and the experimental flow-size distribution of any traffic that will be sent through, and monitored in the metrology platform of Grid5000.

\section{Rate limitation mechanisms}

The last major aspect of the experimentation is the careful design of traffic generation. In real networks, flows are not the fluid ON/OFF flows of the $M / G / N$ model: packets composing the flows are sent entirely, one after the other, at the wire bit-rate. This acts as an ON/OFF sending process. Following on, a critical feature to consider in network experiment design, is the mechanism of traffic generation, especially the rate at which the packets are sent. An important parameter is the aggregation level of the traffic $K$, defined as the ratio between the bottleneck capacity $C$ and the access link nominal capacity $C_{a}$. In xDSL context and more generally in the Internet, it is not rare to have $\mathrm{K}$ ranging over 1000 , while in the data-center context, $\mathrm{K}$ is around 1 or 10 . In our Grid5000 setup the $\mathrm{K}$ factor is close to 1 . To obtain a $\mathrm{K}$ factor larger than 100 (so as to mimic the natural rate limit based upon the bandwidth of the users' uplink in the Internet configuration) and to insure an aggregated throughput average lower than $C=1 \mathrm{~Gb} / \mathrm{s}$, the sources rate has to be limited at most to $10 \mathrm{Mb} / \mathrm{s}$.

End-host based mechanisms can control the individual flows in a scalable and simple way [33]. When considering fixed size packets, the way to modify data rates over a large period 
of time is to vary inter-packets intervals. To calculate these intervals, one considers the time source that can be used to enforce the limitation. In end-host systems, four different time sources are available: a) userland timers, b) TCP self clocking namely RTT of the transfer's path, c) OS's kernel timers, d) packet-level clocking. In our experiments we used three rate limitation approaches which act at different time scales: the first one is based on packet-level clocking (packet spacer), the second one on OS's kernel timers (Token Bucket), the last one on TCP self clocking namely RTT of the transfer's path (window size limitation).

The first two methods rely on the linux traffic shaping mechanism: with the tc utility [34], the qdisc associated to a network interface (the queue in which packets are put before being sent to the network card) is configured. The PSP (PSPacer) [35] qdisc spaces packets by inserting IEEE 802.3x PAUSE packets. These PAUSE packets are discarded at the input port of the first switches. With this mechanism, packets are regularly spaced and short bursts are avoided. The second method resorts to HTB (Hierarchical Token Bucket) qdisc [36] that uses a bucket constantly filled by tokens at the configured target rate. With this qdisc the average rate limit can be overridden during short bursts.

The third and last method modifies the TCP window size to slow down the throughput. The formula window_size = target_throughput $\times R T T$ determines the TCP window size to use to limit the sending rate to target_throughput. This mechanism works well if the window size is not to small, which means also that the target throughput and/or the RTT should not be too small either. As the TCP limitation acts for each TCP connection, many sources located on the same node can have independent rate limitation which is not the case for qdisk-based limitation mechanisms. To limit the rate of a $1 \mathrm{~Gb} / \mathrm{s}$ source to $5 \mathrm{Mbps}$ with full size (1500 Bytes) Ethernet packet and RTT of $12 \mathrm{~ms}$ one has to fix the window size to $7.5 \mathrm{kBytes}$ (corresponding to 5 full-size Ethernet packet).

\section{Experiments description}

Using the facilities offered by Grid5000, our metrology facilities and the rate control mechanisms for traffic generation, several experiments were performed, and we elaborate here on their rationale. First, the general experimental conditions are presented.

The primary interest here is the effect of flow size distributions on self-similarity, when each client behaves like a ON/OFF source model, where a ON period corresponds to a flow emission, and a OFF period to a silent source. The ON (respectively the OFF) lengths are random variables drawn independently and identically distributed, following the specific probability distribution $P_{\mathrm{ON}}$ (respectively $P_{\mathrm{OFF}}$ ) we want to impose on the flow durations $\tau_{\mathrm{ON}}$ (respectively, on the silent periods, $\left.\tau_{\mathrm{OFF}}\right)$. The emission of packets in each flow is controlled by one of the methods described in previous Section, each source rate being limited to $5 \mathrm{Mb} / \mathrm{s}$ to avoid congestion at the $1 \mathrm{~Gb} / \mathrm{s}$ bottleneck.

All experiments consist of one trace of 8-hour traffic generation, representing a total of approximately $n=5.10^{6}$ flows.

\begin{tabular}{|c||c|}
\hline & description \\
\hline \hline Client nodes & Sun Fire V20z (bi-opteron) \\
Kernel & GNU/Linux 2.6.18.3 \\
TCP variant & Bic, with SACK \\
iperf version & 2.0 .2 \\
\hline Topology & Butterfly \\
Bottleneck & $1 \mathrm{~Gb} / \mathrm{s}$ \\
$R T T$ & $12 \mathrm{~ms}$ \\
Sources nb. & 100 \\
Source rate & $5 \mathrm{Mb} / \mathrm{s}$ \\
\hline Exp. duration & $8 \mathrm{hours}$ \\
Flows nb. & $5.10^{6}$ \\
Aggregation time & $\Delta=100 \mu \mathrm{s}$ \\
Flow timeout & timeout $=100 \mathrm{~ms}$ \\
\hline
\end{tabular}

TABLE I

FIXED EXPERIMENTAL GLOBAL PARAMETERS.

As explained before, flows are reconstructed from the traces and we extract their flow sizes (in packets) $W=\left\{w_{i}, i=\right.$ $1, \ldots, n\}$, as well as the OFF times series corresponding to the inter-flow times for each source. Grouping and counting the packets in each contiguous time interval of width $\Delta=100$ $\mu \mathrm{s}$, yields the aggregated traffic time series $X^{(\Delta)}(t)$.

In order to clearly define the terms of application of Taqqu's Theorem on real traffic traces, as well as to identify possible interactions with other factors, we designed four series of experiments whose parameters are summarized in table II.

Experiment A: This is the cornerstone experiment to check relation (14). Distribution of the $\mathrm{ON}$ periods are prescribed to Pareto laws with mean $\mu^{\mathrm{ON}}=0.24 \mathrm{~s}$ (corresponding to a mean flow size of $\langle P\rangle=100$ packets). The experiment is performed ten times with different prescribed tail index $\alpha_{\mathrm{ON}}$, varying from 1.1 to 4 . OFF periods are kept exponentially distributed with mean $\mu^{\mathrm{OFF}}=\mu^{\mathrm{ON}}$. For each value of $\alpha_{\mathrm{ON}}$, an experimental point $\left(\widehat{\alpha}_{\mathrm{ON}}, \widehat{H}\right)$ is empirically estimated. Moreover, to evaluate the possible influence of the protocol, and of the workload generation mechanism, the same series of experiments is reproduced with TCP (window size limitation) and with UDP (user-level packet pacing) first, and then using PSP, HTB and TCP throughput controls. The exact same trial of random variables defining the flow lengths is used for all experiments that imply the same probability law $P_{\mathrm{ON}}$.

Experiment B: Under similar conditions as in series A, the mean of the ON periods takes on two different values $\mu^{\mathrm{ON}}=$ $0.24 \mathrm{~s}$ and $\mu^{\mathrm{ON}}=2.4 \mathrm{~s}$, corresponding to mean flow sizes $\langle P\rangle=100$ and $\langle P\rangle=1000$ packets, respectively. The objective is here to relate $\langle P\rangle$ to the lower scale bound $\Delta_{j_{1}}=2^{j_{1}} \Delta$ defining a sensible regression range to estimate $H$.

Experiment C: The protocol (TCP), the throughput limitation mechanism (TCP window limitation) and the mean flow size $(\langle P\rangle=1000)$ being fixed, we investigate now to role of the OFF periods distribution on the self-similar exponent $H$. Distribution of the OFF periods are prescribed to Pareto laws with mean $\mu^{\mathrm{OFF}}=2.4 \mathrm{~s}$. The experiment is repeated with different prescribed tail index $\alpha_{\mathrm{OFF}}$, varying from 1.1 to 4. ON periods are kept exponentially distributed with mean $\mu^{\mathrm{ON}}=\mu^{\mathrm{OFF}}$. For each value of $\alpha_{\mathrm{OFF}}$, an experimental point 


\begin{tabular}{|c|c|c|c|c|c|c|}
\hline & Proto & $\begin{array}{c}\text { Band } \\
\text { lim }\end{array}$ & $\alpha_{\mathrm{ON}}$ & $\alpha_{\text {OFF }}$ & $\langle P\rangle$ & $\begin{array}{l}\text { meas } \\
\text { param }\end{array}$ \\
\hline \multirow[t]{2}{*}{ A } & TCP & $\begin{array}{l}\text { PSP } \\
\text { HTB } \\
\text { TCP }\end{array}$ & \multirow[t]{2}{*}{$1.1-4$} & \multirow[t]{2}{*}{ - } & \multirow[t]{2}{*}{100} & \multirow[t]{2}{*}{$\begin{array}{l}\widehat{H} \\
\widehat{\alpha}\end{array}$} \\
\hline & UDP & iperf & & & & \\
\hline B & TCP & TCP & $1.1-4$ & - & $\frac{100}{1000}$ & $\Delta_{j_{1}}^{\langle P\rangle}$ \\
\hline C & TCP & TCP & - & $1.1-4$ & 1000 & $\widehat{H}$ \\
\hline \multirow[t]{2}{*}{ D } & TCP & TCP & \multirow{2}{*}{$1.1-4$} & \multirow[t]{2}{*}{ - } & \multirow[t]{2}{*}{100} & \multirow{2}{*}{$\widehat{h}_{\mathrm{loc}}$} \\
\hline & UDP & iperf & & & & \\
\hline
\end{tabular}

TABLE II

EXPERIMENTAL CONDITIONS SUMMARY.

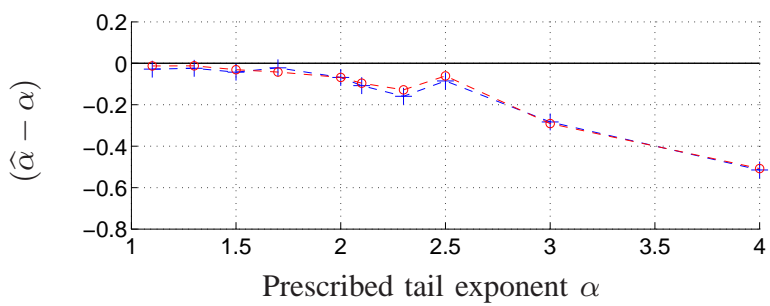

Fig. 3. Difference between the prescribed HT index $\alpha$ and the actually estimated HT index $\widehat{\alpha}$ for the set of different values of $\alpha$ used in experiment series A (see Tab. II) for two different protocols: TCP (+) and UDP (o).

$\left(\widehat{\alpha}_{\mathrm{OFF}}, \widehat{H}\right)$ is empirically estimated.

Experiment D: The last series of experiments aims at investigating self-similarity at finer scales (lower than the RTT scale), and whose origin is distinct from long range dependance phenomena. The variable parameter is the tail index as in experiment $\mathrm{A}$, yet the scaling law index will be estimated in the short time-scales limit, in order to characterize the traffic burstiness from the process $X^{(\Delta)}$. Under the same experimental conditions as in series $\mathrm{A}$, we then evaluate how the protocols (TCP versus UDP) entail a significant change in the traffic burstiness.

Specifically for series A and C, where ON and OFF periods are statistically forced, it is crucial for those experiments to guarantee a zero loss traffic. Otherwise, flow lengths and/or silence periods may deviate from the prescribed distributions due either to packet drop and re-emission, or to exponential back-offs.

\section{RESUlTS AND DISCUSSION}

\section{A. Verifying Taqqu's relation}

For every trace, we use the wavelet-based methodologies described in Section III for heavy tail and self-similarity analyses. The estimated tail index $\hat{\alpha}$ (corresponding to either $\widehat{\alpha}_{\mathrm{ON}}$ or $\widehat{\alpha}_{\mathrm{OFF}}$, clear from the context of Tab. II) results from the linear regression of Eq. (11) applied to the flow size sequence $W$ (or the OFF times series), where a sixth order derivative of a Gaussian wavelet is systematically used. The self-similarity index $\hat{H}$ is estimated from the LD plots of the aggregated time series $X^{(\Delta)}$, using a standard Daubechies wavelet with 3 vanishing moments [23].
1) Tail index estimates: Proceeding with experiment $\mathrm{A}$, for different values of the tail index of the flow size distribution, Fig. 3 displays the differences between the prescribed value $\alpha$ and the actually estimated value $\widehat{\alpha}$. The two experimental curves, corresponding to TCP and UDP protocols respectively, superimpose almost perfectly. Beyond coherence with the fact that the exact same trial of random variables defining the flow lengths is used in both cases, such a concordance demonstrates that the flow reconstruction procedure, for both TCP or UDP packets grouping, is fully operative, notably including a relevant timeout adjustment (t imeout $=100$ $\mathrm{ms})$.

Fig. 3 also shows an increasing difference $(\widehat{\alpha}-\alpha)$ with $\alpha$. In our understanding, this is not caused by an increasing bias of the HT estimator, which is known to perform equally well for all $\alpha$ values. It is rather caused by the natural difficulty to prescribe large values of $\alpha$ over fixed duration. Indeed, as $\alpha$ increases, large flows become more rare, and the number of observed elephants during the constant duration ( 8 hours) of the experiments naturally decreases, then deviating from a statistically relevant sample. This observation is fully consistent with arguments developed in [37]. Notwithstanding this satisfactory agreement, in the sequel we will systematically refer to $\widehat{\alpha}$ rather than to the prescribed $\alpha$.

2) Gaussianity: Before verifying the presence of long range dependence in the aggregated traffic time series, we first need to validate the normal assumption (recall that the increment process of a $\mathrm{fBm}$ is a stationary and Gaussian process). Thus, for each trace described in Tab. II, the Kurtosis index ${ }^{2}$ of the aggregated traffic time series distribution was computed at several different aggregation intervals. As, the Kurtosis index was always found to lie between 3.0 and 3.1, for aggregation intervals larger than $\Delta=10 \mathrm{~ms}$, we conclude that the aggregated traffic time series is a reasonably Gaussian process beyond this limit.

3) LD-description: Fig. 4 shows typical LDs of aggregated traffic time series, obtained under similar conditions (experiment series A of Tab. II, TCP protocol, TCP window limitation), for 4 different values of $\alpha$. Such plots enable a generic phenomenological description of LDs: 3 different ranges of scales can be visually identified, whose bounds do not seem to drastically vary with $\alpha$ :

Coarse scales: In the coarse scale domain, a clear scaling behavior is systematically observed. As mentioned earlier, Taqqu's Theorem relates heavy tails and self-similarity in the asymptotic limit of coarse scales. Therefore, the scaling exponent at coarse scales, denoted $\widehat{H}$, is a candidate to match that involved in relation (14).

Fine scales: At fine scales, another clear scaling behavior is also observed. However, the corresponding scaling index, denoted $h$, is no longer related to Taqqu's Theorem prediction but rather to a local regularity property of the data.

Medium scales: Intermediate scales mostly connect the two scaling behaviors happening for fine and coarse scales, but exhibit no noticeable scaling behavior.

\footnotetext{
${ }^{2}$ The Kurtosis index of a R.V. is defined as the ratio of its fourth order moment over its square variance, and takes on the value 3 in the normal case.
} 


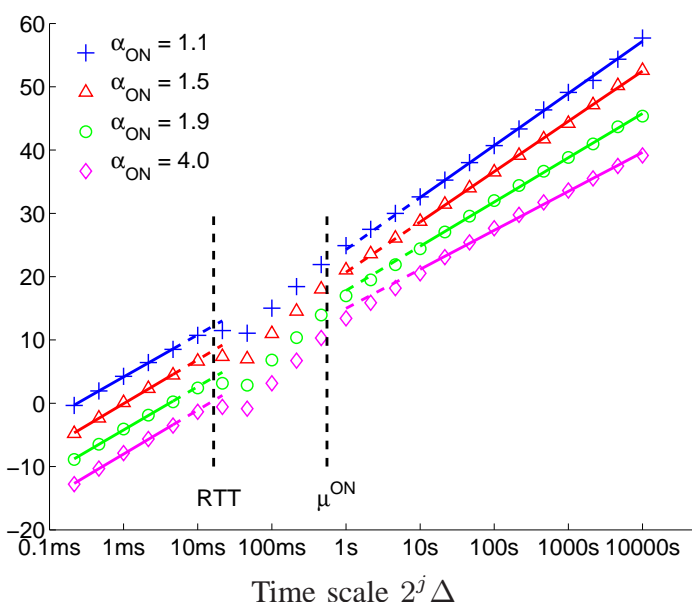

Fig. 4. Wavelet $\log$-diagrams $\log S(j)$ versus time scale $j$ of aggregated traffic (aggregation interval $\Delta=100 \mu \mathrm{s}$ ). Log-diagrams correspond to 4 time series obtained under similar experimental conditions with the protocol TCP, with 4 different values of $\alpha_{\mathrm{ON}}: 1.1(+), 1.5(\triangle), 1.9(\circ)$ and $4.0(\diamond)$. For the sake of readability, curves were vertically shifted to avoid overlapping.

In Fig. 4, vertical lines materialize the two transition scales between the three depicted domains and can hence be identified as characteristic time scales of the data. Let us now investigate the nature of these characteristic times.

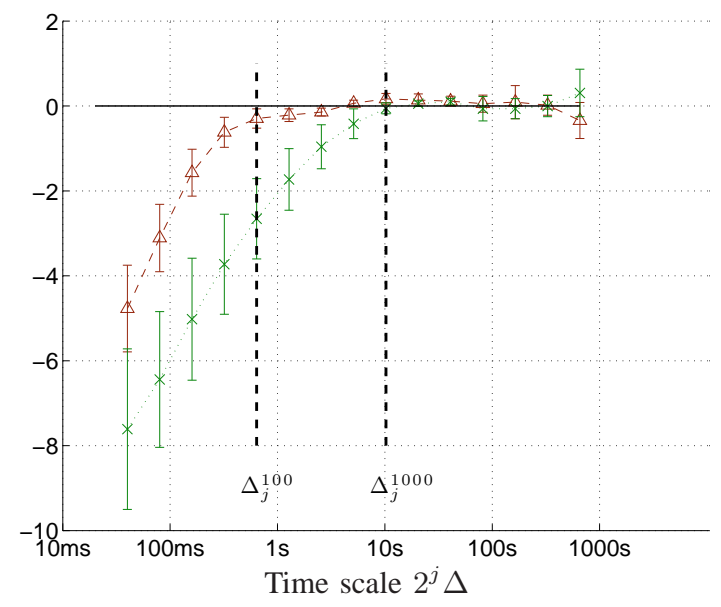

Fig. 5. Averaged normalized log-diagrams for two different mean sizes $\langle P\rangle$ of the transmitted flows: $(\times)\langle P\rangle=1000$ packets $-(\triangle)\langle P\rangle=100$ packets.

4) Coarse scales domain lower bound: It is alluded in [21] that the range of scales where self-similarity can be measured is beyond a characteristic scale, referred to as the knee of the LD, and that it is essentially controlled by the mean flow duration. To investigate this argument in the context of our analyses, we designed two experiments series with two different values of the mean flow duration (series B of Tab. II). For each case, all the LDs corresponding to the different values of $\alpha$ are computed. To emphasize the impact of the mean flow duration, we substracted to each $L D$, the asymptotic linear trend, obtained by linear regression between a scale $\Delta_{j_{1}}$, clearly above the knee position, and the maximum available scale $\Delta_{j_{\max }}$. Fig. 5 shows, both for $\langle P\rangle=100$ and $\langle P\rangle=1000$ the mean and standard deviation over all

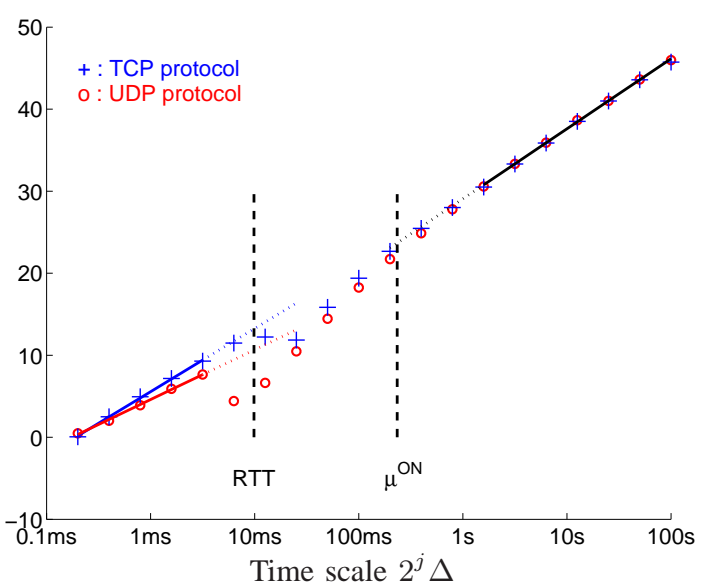

Fig. 6. Wavelet $\log$-diagrams $\log S(j)$ versus time scale $j$ of aggregated traffic (aggregation interval $\Delta=100 \mu \mathrm{s}$ ). Log-diagrams correspond to two time series obtained under similar experimental conditions, for $\alpha_{\mathrm{ON}}=1.5$, with two different protocols: TCP (+) and UDP (o).

normalized LDs. Each graph clearly exhibits a slope break: at scale $\Delta_{j}^{100}=0.64 \mathrm{~s}$ when $\langle P\rangle=100$ and at scale $\Delta_{j}^{1000}=10.28 \mathrm{~s}$ when $\langle P\rangle=1000$. Although for $\langle P\rangle=100$, the knee effect slightly smoothes out, the linear behavior observed for $\langle P\rangle=1000$ clearly extends with the same slope beyond $\Delta_{j}^{1000}$ up to $\Delta_{j}^{100}$. Unquestionably, the measured knee position undergoes the same variations as the mean flow duration, both quantities being in the same order of magnitude: $\Delta_{j}^{100} \simeq \mu_{100}^{\mathrm{ON}}(0.24 \mathrm{~s})$ and $\Delta_{j}^{1000} \simeq \mu_{1000}^{\mathrm{ON}}(2.4 \mathrm{~s})$. This analysis confirms the intuition that the coarse scale range, where selfsimilarity is to be measured, lies above the knee of the LD, whose position is in the same order of magnitude as the mean flow duration. The coarse scales can then be renamed: the flow scales, or the file scales.

5) Protocol, rate limitation and coarse scales: As we investigate Taqqu's relation, we now focus on the coarse scale domain. To inquire on the impact of the protocol on the coarse scales, Fig. 6 shows the LDs obtained with two different protocols : TCP and UDP (for $\alpha=1.5$ ). Fig. 6 evidences the central feature that both LDs are undistinguishable in the coarse scale domain. We conclude that, when source rate limitation precludes congestion, the protocol has no impact on the coarse scale SS.

Similarly, to inquire on the impact of the rate limitation mechanism on the coarse scales, Fig. 7 shows typical LDs ( $\alpha=1.5$, TCP) obtained with three different rate limitation mechanisms: PSP, HTB and TCP window limitation. As the three LDs cannot be distinguished one from the other in the coarse scale domain, we conclude that the rate limitation mechanism has no influence on the scaling behavior at coarse scales.

6) $H$ versus $\alpha_{\mathrm{ON}}$ : Practically, to perform an empirical validation of Eq. (14), we need to estimate the scaling parameter $H$ and thus to carefully choose the range of scales where the regression is to be performed. Although the knee position has been related to a measurable experimental parameter (the mean flow duration), a systematic choice of the regression range at coarse scales would certainly be hazardous. Instead, we defined for each trace an adapted regression range, based on a 
(a)

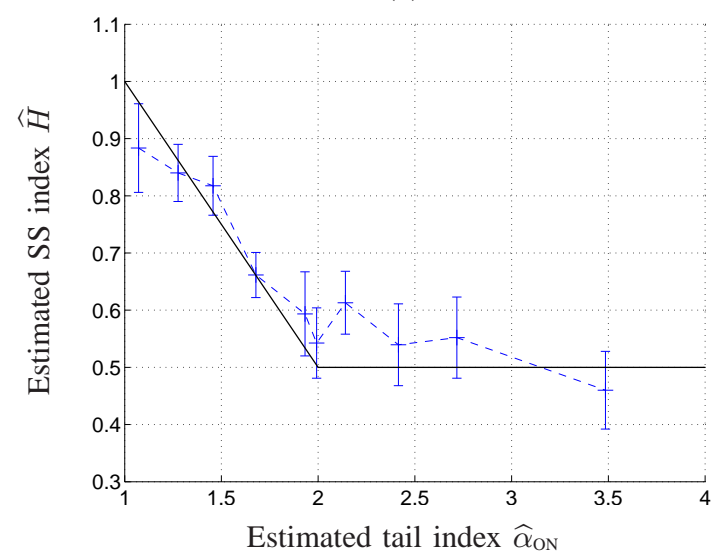

(b)

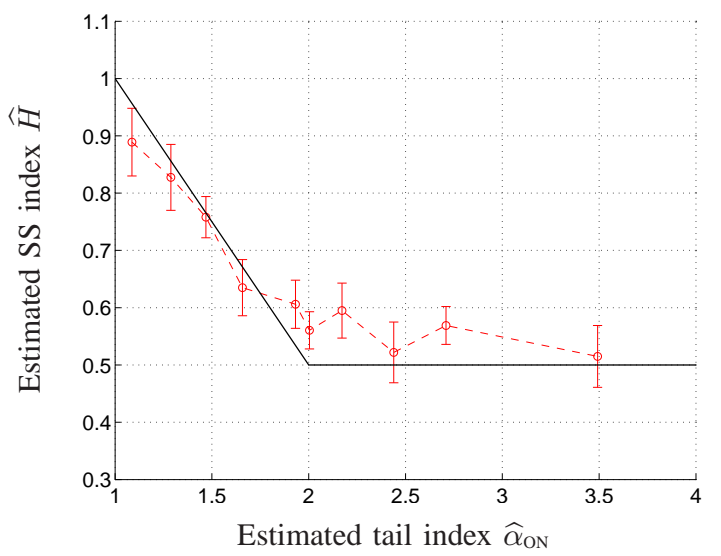

Fig. 8. Estimated Self-Similar index $\widehat{H}$ of the aggregated traffic (aggregation interval $\Delta=100 \mu \mathrm{s}$ ) versus estimated tail index $\widehat{\alpha}_{\mathrm{ON}}$ of the corresponding flow size distribution. Solid plots represent the theoretical model of relation (14), dashed plots correspond to experimental results: (a) with TCP protocol; (b) with UDP protocol.

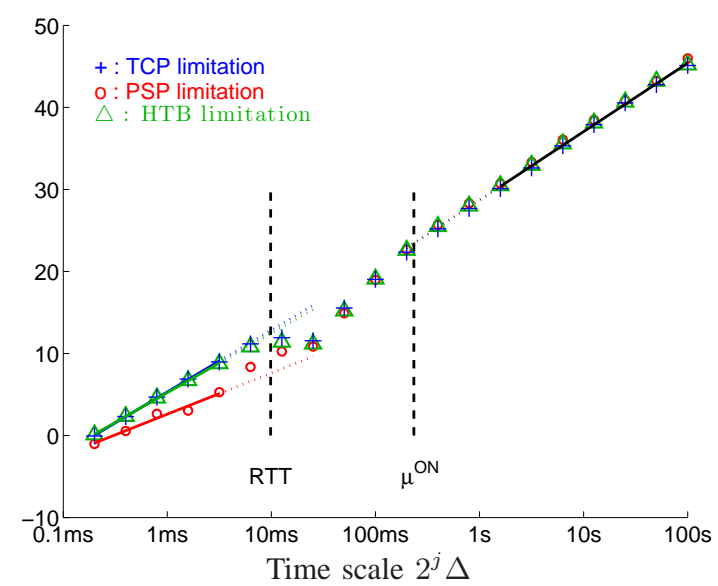

Fig. 7. Wavelet $\log$-diagrams $\log S(j)$ versus time scale $j$ of aggregated traffic (aggregation interval $\Delta=100 \mu \mathrm{s}$ ). Log-diagrams correspond to 3 time series obtained under similar experimental conditions, for $\alpha_{\mathrm{ON}}=1.5$, with three different rate limitation mechanisms: TCP $(+), \operatorname{PSP}(\circ)$ and HTB $(\triangle)$.

linearity criterion, and found that all regression ranges defined like this, encompass a scale interval $\left(\max _{\alpha} \Delta_{j_{1}}=20.5 \mathrm{~s}\right.$ and $\Delta_{j_{\max }}=1310 \mathrm{~s}$ ), significantly extended to warrant statistically reliable SS exponent estimates.

Fig. 8 plots the estimates of coarse scale SS exponents against those of the HT indices. Confidence intervals for $\widehat{H}$ displayed on the graphs are supplied by the estimation procedure detailed in Section III-A2 [6], [22] (recall that normal hypothesis underlying the estimation of these confidence intervals was successfully verified on our data). Such estimations are conducted independently for TCP and UDP protocols. For both protocols, estimations show a very satisfactory agreement with Taqqu's Theorem prediction. To the best of our knowledge, this theoretical relation between selfsimilarity and heavy tail had never been observed with such a satisfactory accuracy, (over a large and significant range of $\alpha$ values). For instance, and although no definitive interpretation has been proposed yet, the offset below the theoretical relation for $\alpha$ close to 1 , and the offset above the horizontal line for $\alpha$ larger than 2 have been drastically reduced when compared to similar analyses results reported in the literature (cf. e.g., [11]). This accuracy results from a number of factors: Firstly, the statistical tools for estimating $H$ and $\alpha$ are chosen amongst the most recent and robust (notably the proposed estimator for $\alpha$ had never been applied before to Internet data); Secondly, the asymptotic coarse scale nature of Taqqu's Theorem is really accounted for by performing estimation in the limit of really coarse scales; Thirdly, this is made possible thanks to the use of really long duration, stationary and controlled traffic time series, wich is enabled by the use of Grid5000 platform.

Additionally, our analyses do confirm that TCP and UDP protocols do not impact this relation, at least under congestion avoidance conditions corresponding to our experimental setup. This is in clear agreement with the findings reported in [12], or [10], showing that TCP is not responsible for the observed self-similarity. However, despite these earlier results, a non negligible number of contributions debated, investigated and argued in favor of an impact of protocols on self-similarity. Our analyses clearly show that the range of scales where protocols impact the LD is far below the characteristic time scales involved in self-similar phenomena.

As long as we actually consider coarse scales (larger than the mean duration of a flow), the only cause for self-similarity is the heavy tail in the flow sizes distribution.

7) OFF periods: To complement the experimental study of Taqqu's Theorem, the experiments of series C (see Tab. II) were designed to assess the influence of heavy-tailed distributed OFF periods on the coarse scale SS exponent $H$. Under experimental conditions detailed in Tab. II, Fig. 9 displays the estimated coarse scale SS exponents against those of the OFF periods HT indices. Since we previously validated that the protocol has no influence on the scaling behavior in coarse scales, these experiments were only performed with TCP protocol.

In contrast to similar results reported in the literature (cf. e.g., Fig. 5 (right) of [11], where the estimated value of $H$ is less than 0.7 , even for $\alpha_{\mathrm{OFF}}=1.05$ ), our results show a perfect 


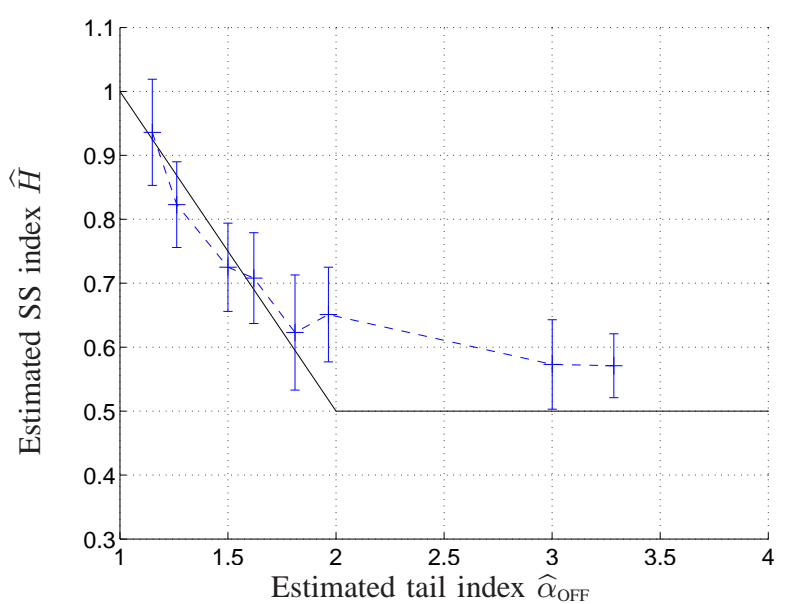

Fig. 9. Estimated Self-Similar index $\widehat{H}$ of the aggregated traffic (aggregation interval $\Delta=100 \mu \mathrm{s}$, TCP) versus estimated tail index $\widehat{\alpha}_{\mathrm{OFF}}$ of the corresponding OFF periods distribution. Solid plots represent the theoretical model of relation (14), dashed plots correspond to experimental results.

agreement with the theoretical relation of Eq. (14). Reasons for this theoretical accord certainly lies in the same origins as the ones evoked in the previous Paragraph (i.e. robust estimators, long-duration stationary traces and controlled configurations), but possibly also, in the fact that we scrupulously avoided congestion, hence statistical alteration of the OFF periods. To the best of our knowledge, this other part of Taqqu's Theorem had never been satisfactorily addressed.

Finally, let us notice that confidence intervals displayed in Fig. 9 are significantly larger than the ones of Fig. 8. This is due to the difficulty of imposing short OFF intervals that led us here to increase the mean duration $\mu_{\mathrm{OFF}}=\mu_{\mathrm{ON}}=2.4 \mathrm{~s}$ (instead of $0.24 \mathrm{~s}$ ). In accordance with the interpretation of Fig. 5, the coarse scale regression range is then consequently reduced.

\section{B. Further analyses of the $L D$}

In previous Section, we focused on the coarse scales of LDs. Let us now turn to the medium and fine scales and study the influence of protocols and rate limitation mechanisms.

1) Medium scales: Firstly, let us notice that, while the mean flow duration gives an upper bound for the medium scale domain, RTT (12 ms) seems to correspond to its lower bound. Therefore, this medium scale range will be referred to as the RTT-scales. Although no scaling behavior is visible in this medium scale range, Fig. 6 shows a significant difference between the LDs obtained from TCP and UDP traffic. This is an expected result as $R T T$ is the characteristic time of action of TCP protocol.

Fig. 7 shows that there is no significant difference in this domain between the LDs corresponding to the three different rate limitation mechanisms. The characteristic time of action of the rate limitation is the mean inter-packet time. Due to the source rate limitation at $5 \mathrm{Mb} / \mathrm{s}$ achieved with 1500-Bytes packets, the mean inter-packet time for one source is $2.4 \mathrm{~ms}$. As the mean number of sources emitting simultaneously is 50 , the mean inter-packet time is $48 \mu \mathrm{s}$, which is much lower than $R T T$. Accordingly, the rate limitation does not impact the traffic at $R T T$ scales.

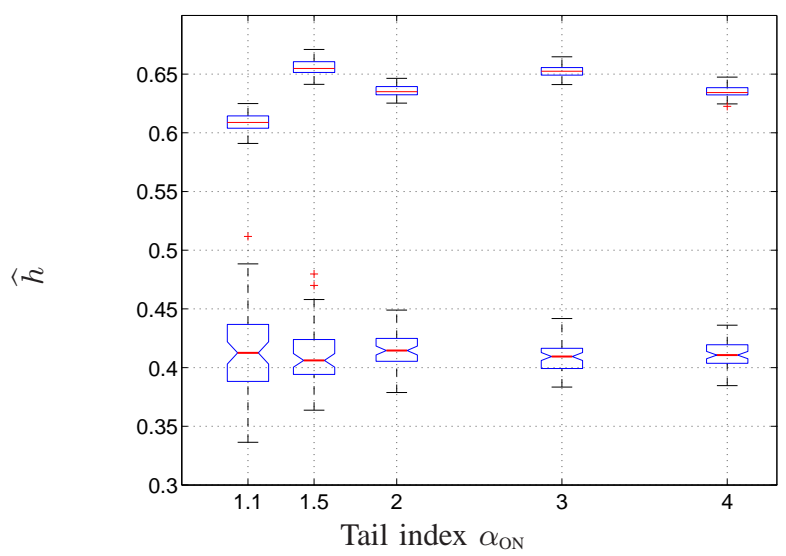

Fig. 10. Fine scale scaling exponent $h$ estimates on aggregated traffic time series $(\Delta=100 \mu s)$. For different values of the tail index $\alpha_{\mathrm{ON}}$ governing the flow size distributions, $h$ is estimated by linear regression of Log-diagrams (see Fig. 6) over the scale range $[0.2-5] \mathrm{ms}$. Notched box-plots correspond to UDP protocol, regular box-plots to TCP protocol.

2) Fine scales: TCP and UDP impact on fine scales scaling. Figure 6 shows a good scaling behavior at fine scales, with a scaling index which seems to be different for UDP and TCP.

To analyze in more details the fine scales scaling exponent, each 8-hour trace corresponding to a particular value of $\alpha$ (see experimental conditions of Experiment A in Table II) are chopped into 66 short-length of duration $T=100 \mathrm{~s}$ each. The resulting time series are then analyzed independently and a fine scaling exponent $h$ estimated. Hence, based on these 66 values of $h$, box-plots are displayed on Figure 10 for each theoretical value of $\alpha$. The values for TCP remain roughly constant around $h \simeq 0.63$. Likewise for UDP, $h$ does not seem to depend on $\alpha$, but stands around 0.4 , a significantly smaller value than that for TCP.

Smaller than RTT, these fine scales correspond to the packet-scales. Clearly then, the scaling index at these scales is sensitive to the packet sending mechanism. When using UDP, packets are emitted individually, separated by an interpacket interval $(2.4 \mathrm{~ms})$ imposed by iperf to maintain the rate limitation ( $5 \mathrm{Mb} / \mathrm{s}$ ). Therefore, UDP traffic is constantly and erratically varying. When using TCP, packets are sent by bursts containing up to 5 packets. Then TCP traffic is bursty, but also sparse, with "long" periods of no packets. We believe that this packet sending scheme difference, in close relationship with our experimental condition (source rate limitation used to avoid congestion) is sole responsible for the observed difference between TCP and UDP on the local regularity.

Bandwidth limitation impact on fine scales scaling. Figure 7 shows that the scaling index at fine scales is approximately the same with TCP and HTB limitation, but it is very different with PSP limitation. This difference can again be explained by the packet sending mechanism. When using HTB limitation, packets are sent by bursts, in the same way as with TCP limitation. This explains why the local regularity observed 
with HTB is the same as the one observed with TCP window limitation. On the contrary, when using PSP, packets are sent individually, in the same way as with UDP. Then, at fine scale, the scaling index observed with PSP is lower than the one observed with TCP limitation, as was the one observed with UDP.

\section{CONCLUSIONS AND PERSPECTIVES}

In this paper, we experimentally demonstrated that the traffic generated on a real network platform conforms to the theoretical bond between the file sizes distribution and the self-similar nature of instantaneous throughput measured at the link level. This work is based on three important innovative factors: the use of accurate estimation tools, a deeper analysis of Taqqu's Theorem applicability conditions and the use of a large scale reconfigurable experimental facility. The wavelet based estimation procedure for $H$ is known as a state-of-theart tool, being one of the most reliable and robust (against non stationarities). Here, it has been used with most care. The $\alpha$ index estimation procedure used here, shown to outperform previous available techniques, had never been used before in the context of Internet data. Widely reckoned, the deeply asymptotic nature of Taqqu's Theorem has been better accounted for by conducting estimations of the self-similarity parameter at really coarse scales (coarse quantifying scales that lie far beyond the system dynamic). This asymptotic limit requires to produce traffic with particularly long observation duration, yet stationary, and well controlled. The nation wide and fully reconfigurable Grid5000 instrument enables generation, control and monitoring of a large number of finely controlled transfer sessions with real transport protocol stacks, end-host mechanisms, network equipments and links. Given such realistic and long traces, we have been able to demonstrate experimentally, and with an accuracy still never achieved with real data nor with simulations, that Taqqu's Theorem and the relation between self-similarity and heavytailness actually holds. In particular, we obtained a significant agreement between theoretical and experimental values at the transition points: $\alpha=2$. This is a particularly involved case, for it mixes difficulties of different kinds regarding the estimation of both $H$ and $\alpha$ indices. Pursuing our empirical certification of Taqqu's relation, we rigorously demonstrated that when the distribution of the OFF periods has an heavier tail than that of flows' size, it prevails at imposing long range dependence to the aggregated traffic. As it is elegantly tackled in [38] and [39], we believe that a precise statistical characterization of idle times is an important factor of traffic modeling. In this direction, we are currently exploiting the flexibility of our original Grid5000 testbed to study how the response of protocol mechanisms to congestion creates new OFF periods, and how these latter do impact the self-similarity properties of traffic.

Following up the controversial discussion about the relationship between transport protocols and self-similarity - raised after and despite Crovella et al.'s meaningful contributions [3] (see e.g. discussion in [12]) - our observations confirm that in loss-free situations, protocols, bitrate mechanisms or packet and flow-level controls do not impact the observed long range dependence. Our analyses show that this is so, because the ranges of scales (segmented according to the $R T T$ and to the mean flow duration) related to self-similarity are far coarser than those (fine and medium scale) associated to such mechanisms. As a natural sequel of the present work, we now plan to confront these results to more actual situations involved in real network applications. In particular, we will generate long-term stationary traces under various congestion and aggregation levels, with heterogeneous source rates, involving different source protocols, mixing variable $R T T$ s, including several bottleneck and buffer capacities and ran with with variants of the high speed transport protocols. A precise study of the self-similarity impact on buffer utilization, queueing delays and dynamics, would certainly be worth investigating as well. In our opinion though, the present study builds the definitive support to get new insights on the relevance of Taqqu's relation regarding actual applications, and eventually, to help researchers design the future transport and network control protocols.

\section{REFERENCES}

[1] V. Paxson and S. Floyd, "Wide area traffic: The failure of Poisson modeling," in SIGCOMM, New York, NY, USA, 1994, pp. 257-268, ACM Press.

[2] W. E. Leland, M. S. Taqqu, W. Willinger, and D. V. Wilson, "On the self-similar nature of ethernet traffic (extended version)," ACM/IEEE Trans. on Net., vol. 2, no. 1, pp. 1-15, Feb. 1994.

[3] M. E. Crovella and A. Bestavros, "Self-similarity in World Wide Web traffic: Evidence and possible causes," IEEE/ACM Trans. on Net., vol. 5, no. 6, pp. 835-846, Dec. 1997.

[4] T. Karagiannis, M. Molle, and M. Faloutsos, "Long-range dependence ten years of internet traffic modeling," IEEE Internet Computing, Sept. 2004.

[5] P. Abry, R. Baraniuk, P. Flandrin, R. Riedi, and D. Veitch, "Multiscale network traffic analysis, modeling, and inference using wavelets, multifractals, and cascades," IEEE Sig. Proc. Magazine, vol. 3, no. 19, pp. 28-46, May 2002.

[6] P. Abry, P. Flandrin, M.S. Taqqu, and D. Veitch, "Wavelets for the analysis, estimation and synthesis of scaling data," in Self-Similar Network Traffic and Performance Evaluation, Kihong Park and Walter Willinger, Eds. 2000, John Wiley \& Sons, Inc.

[7] M. S. Taqqu, W. Willinger, and R. Sherman, "Proof of a fundamental result in self-similar traffic modeling," SIGCOMM CCR, vol. 27, no. 2, pp. 5-23, 1997.

[8] W. Willinger, M. S. Taqqu, R. Sherman, and D. V. Wilson, "Selfsimilarity through high-variability: statistical analysis of ethernet lan traffic at the source level," IEEE/ACM Trans. on Net., vol. 5, no. 1, pp. 71-86, 1997.

[9] P. Doukhan, G. Oppenheim, and M.S. Taqqu, Long-Range Dependence: Theory and Applications, Birkhäuser, Boston, 2003.

[10] L. Guo, M. Crovella, and I. Matta, "Corrections to 'How does TCP generate pseudo-self-similarity?'," SIGCOMM CCR, vol. 32, no. 2, 2002.

[11] K. Park, G. Kim, and M. Crovella, "On the relationship between file sizes, transport protocols, and self-similar network traffic," in Int. Conf. on Network Protocols, Washington, DC, USA, 1996, p. 171, IEEE Computer Society.

[12] D. R. Figueiredo, B. Liu, A. Feldmann, V. Misra, D. Towsley, and W. Willinger, "On TCP and self-similar traffic," Performance Evaluation, vol. 61, no. 2-3, pp. 129-141, 2005

[13] R. Bolze, F. Cappello, E. Caron, M. Daydé, F. Desprez, E. Jeannot, Y. Jégou, S. Lanteri, J. Leduc, N. Melab, G. Mornet, R. Namyst, P. Primet, B. Quetier, O. Richard, E.-G. Talbi, and I. Touche, "Grid'5000: a large scale and highly reconfigurable experimental grid testbed.," Int. J. of High Performance Computing Applications, vol. 20, no. 4, pp. 481-494, nov 2006.

[14] K. Park and W. Willinger, Self-Similar Network Traffic and Performance Evaluation, John Wiley \& Sons, Inc., New York, NY, USA, 2000. 
[15] P. Barford, J. Kline, D. Plonka, and A. Ron, "A signal analysis of network traffic anomalies," in SIGCOMM Internet Measurement Workshop, Marseille, France, Nov. 2002.

[16] B. White, J. Lepreau, L. Stoller, R. Ricci, S. Guruprasad, M. Newbold, M. Hibler, C. Barb, and A. Joglekar, "An integrated experimental environment for distributed systems and networks," ACM SIGOPS Operating Systems Review, vol. 36, no. SI, pp. 255-270, 2002.

[17] D. G. Andersen, H. Balakrishnan, M. F. Kaashoek, and R. Morris, "Resilient overlay networks," in Proc. of the 18th ACM SOSP, Oct. 2001.

[18] A. Bavier, M. Bowman, B. Chun, D. Culler, S. Karlin, L. Peterson, T. Roscoe, T. Spalink, and M. Wawrzoniak, "Operating system support for planetary-scale network services," in Proc. of the 1st Symposium on Network System Design and Implementation, Mar. 2004.

[19] A. B. Downey, "Evidence for long-tailed distributions in the internet," in SIGCOMM Internet Measurement Workshop, New York, NY, USA, 2001, pp. 229-241, ACM Press.

[20] C. Barakat, P. Thiran, G. Iannaccone, C. Diot, and P. Owezarski, "A flow-based model for internet backbone traffic," in SIGCOMM Internet Measurement Workshop, New York, NY, USA, 2002, pp. 35-47, ACM Press.

[21] N. Hohn, D. Veitch, and P. Abry, "Cluster processes, a natural language for network traffic," IEEE Trans. on Sig. Proc. - Special Issue on Sig. Proc. in Net., vol. 8, no. 51, pp. 2229-2244, Oct. 2003.

[22] P. Abry, P. Gonçalves, and P. Flandrin, "Wavelets, spectrum analysis and $1 / f$ processes," in Lecture Notes in Statistics: Wavelets and Statistics, A. Antoniadis and G. Oppenheim, Eds., 1995, vol. 103, pp. 15-29.

[23] S. Mallat, A Wavelet tour of signal processing, Academic Press, 1999.

[24] R. J. Adler, R. E. Feldman, and M. S. Taqqu, A Practical Guide To Heavy Tails, Chapman and Hall, New York, 1998.

[25] J. H. McCulloch, "Measuring tail thickness to estimate the stable index alpha: A critique," American Stat. Asso., vol. 15, pp. 74-81, 1997.

[26] P. Gonçalves and R. Riedi, "Diverging moments and parameter estimation," J. of American Stat. Asso., vol. 100, no. 472, pp. 1382-1393, December 2005

[27] "Iperf, NLANR/DAST project," http://dast.nlanr.net/Projects/Iperf/.

[28] Y. Kodama, T. Kudoh, T. Takano, H. Sato, O. Tatebe, and S. Sekiguchi, "GNET-1: Gigabit ethernet network testbed," in Proc. of the IEEE Int. Conf. Cluster 2004, San Diego, California, USA, Sept. 20-23 2003.

[29] "Ipsumdump," http://www.cs.ucla.edu/ kohler/ipsumdump/.

[30] N. Duffield, C. Lund, and M. Thorup, "Estimating flow distributions from sampled flow statistics," IEEE/ACM Trans. on Net., vol. 13, no. 5, pp. 933-946, October 2005.

[31] M. E. Crovella, M. S. Taqqu, and A. Bestavros, "Heavy-tailed probability distributions in the World Wide Web," in A Practical Guide To Heavy Tails, Robert J. Adler, Raisa E. Feldman, and Murad S. Taqqu, Eds., chapter 1, pp. 3-26. Chapman and Hall, New York, 1998.

[32] S. I. Resnick, H. Dress, and L. De Haan, "How to make a hill plot," OR \& IE University, 1998.

[33] S. Soudan, R. Guillier, and P. Vicat-Blanc Primet, "End-host based mechanisms for implementing flow scheduling in gridnetworks," in GridNets 2007, Oct. 2007.

[34] "Iproute2, the linux foundation," http://www.linuxfoundation.org/en/Net:Iproute2/.

[35] R. Takano, T. Kudoh, Y. Kodama, M. Matsuda, H. Tezuka, and Y. Ishikawa, "Design and evaluation of precise software pacing mechanisms for fast long-distance networks," in PFLDnet, Lyon, France, 2005.

[36] "Hierachical token bucket packet scheduler," http://luxik.cdi.cz/ devik/qos/htb/.

[37] M. Roughan, J. Yates, and D. Veitch, "The mystery of the missing scales: Pitfalls in the use of fractal renewal processes to simulate LRD processes," in ASA-IMS Conf. on Applications of Heavy Tailed Distributions in Economics, Engineering and Statistics, American University, Washington, DC, June 1999.

[38] L. Guo, M. Crovella, and I. Matta, "How does TCP generate pseudoself-similarity?", in MASCOTS, Washington, DC, USA, 2001, p. 215, IEEE Computer Society.

[39] D. R. Figueiredo, B. Liu, V. Misra, and D. Towsley, "On the autocorrelation structure of TCP traffic," Computer Networks, vol. 40, no. 3, pp. 339-361, 2002.

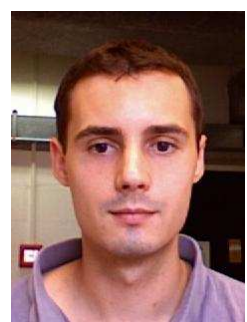

Patrick Loiseau was born in Paris, France, in 1982. He graduated from École Normale Suprieure de Lyon, France, in physics. He received the degree of Professeur-Agrégé de Sciences-Physiques in 2005, and the M.S. degree of physics at ÉNS Lyon, France, in 2006. Presently, he is pursuing a Ph. D. degree at the computer science department of the ÉNS Lyon, France. His main research interests include waveletbased analysis and modeling of scaling phenomena in networks and grid traffic, with application to the Quality of Service. His is also interested in statistical methods application to estimation problems.

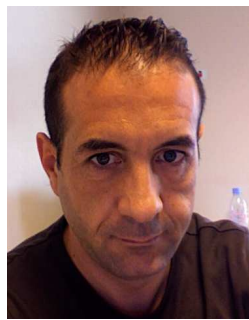

Paulo Gonçalves graduated from the Signal Processing Department of CPE Lyon, France in 1993. He received the M.S. and Ph.D. degrees in signal processing from INPG, France, in 1990 and 1993 respectively. While working toward his Ph.D. degree, he was with ÉNS Lyon. In 1994-96, he was a Postdoctoral Fellow at Rice Univ., US. Since 1996, he is associate researcher at INRIA, first with Fractales (1996-99), then with IS2 (20002003) and now with the team RESO at the Parallel Computing Lab. (LIP), ÉNS Lyon. From 2003 to 2005, he was on leave at IST Lisbon, Portugal.

His research interests are in multiscale analysis and in wavelet-based statistical inference. His principal application is in metrology and deals with grid traffic statistical characterization and modelling for protocole quality assessment and control.

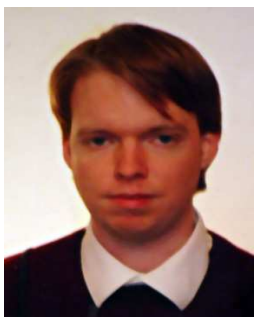

Guillaume Dewaele was born in Hazebrouck, France, in 1978. He made his studies at the École normale supérieure de Lyon, France, receiving the Professeur-Agrégé de Sciences Physiques degree in 2000, a Master in Physics in 2001 and defended a Ph.D. degree in Numerical Simulation and Computer Vision in 2005. Since November 2005, he has been a Agregé Préparateur (Lecturer) with the Laboratoire de Physique, ÉNS Lyon. His research interests are in signal and image processing, numerical simulations and internet traffic measurement and modeling, for anomaly detection and identification purposes

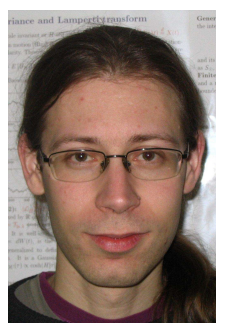

Pierre Borgnat was born in Poissy, France, in 1974. He made his studies at the École Normale Supérieure de Lyon, France, receiving the Professeur-Agrégé de Sciences Physiques degree in 97, a Ms. Sc. in Physics in 99 and defended a Ph.D. degree in Physics and Signal Processing in 2002. In 20032004, he spent one year in the Signal and Image Processing group of the IRS, IST (Lisbon, Portugal) Since October 2004, he has been a full-time CNRS researcher with the Laboratoire de Physique, ÉNS Lyon. His research interests are in statistical signal processing of non-stationary processes (time-frequency representations, time deformations, stationarity tests) and scaling phenomena (time-scale, wavelets) for complex systems (turbulence, networks,...). He is also working on Internet traffic measurements and modeling, especially for security enforcement based on measurements. 


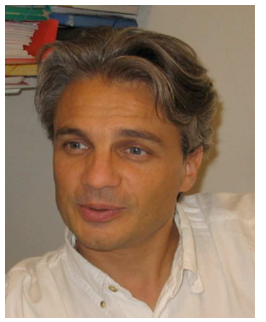

Patrice Abry was born in Bourg-en-Bresse, France in 1966. He is Professeur-Agrégé de Sciences Physiques (1989) and completed a Ph.D. in Physics and Signal Processing in 1994. Since october 95, he is a permanent CNRS researcher, at the Laboratoire de Physique of Ecole Normale Supérieure de Lyon. Patrice Abry received the AFCET-MESRCNRS prize for best Ph.D. in Signal Processing for the years 93-94 and is the author of a book "Ondelettes et Turbulences", Diderot, 1997. Since 2004, he is member of the SPS-SPTM committee. His current research interests include the wavelet based analysis and modeling of scaling phenomena, of hydrodynamic turbulence and computer network teletraffic.

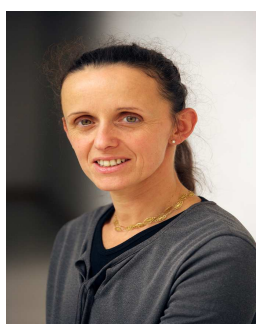

Pascale Vicat-Blanc Primet is senior researcher at the National Institute of Research in Computer Science (INRIA) since 2005. Since 2002, she is leading, the INRIA RESO team (22 researchers and engineers) within the LIP laboratory of the École Normale Superieure de Lyon. Since beginning of 2008, she also leads the "Semantic Networking" research team of INRIA-Bell Labs common laboratory. Her research interests include High Speed and High Performance Networks, Internet protocols design and architecture, Quality of Service, network and traffic measurement, Network programmability and virtualization, Grid networking She is member of the scientifical committee of Grid5000's/ALADDIN - French Computer Science Grid initiative. She has published more than 80 papers in International Journal and Conferences in Networking and Grid computing. She obtained her Habilitation Diriger les Recherches from University of Lyon in 2002, her PhD (88) in Computer Science, MsC (84) and Engineer diploma (84) in CS from INSA de Lyon. 